\title{
DESIGN OF A 2D AHMED BODY REAR SURFACE TO OPTIMIZE DRAG AND DOWNFORCE USING MULTI-OBJECTIVE GENETIC ALGORITHM: NSGA-II
}

\author{
Raphael Aranha ${ }^{1 *}$, Anton Alconl ${ }^{2}$, Kais Abdulmawjood ${ }^{3}$, Martin Angelin-Chaab ${ }^{4}$ \\ ${ }^{1,2,3,4}$ Automotive Mechanical and Manufacturing Department 1, Ontario Tech University, Oshawa, ON, Canada \\ *raphael.aranha@ontariotechu.net
}

\begin{abstract}
In the design of race cars or airplanes the aerodynamics, such as its rear surface, is important to achieve the best performance possible. The objective of this paper was to optimize the rear surface design of a two-dimensional body (2D) to reduce drag and lift (to improve downforce) utilizing a metaheuristic optimization program. Drag and lift shape optimization can be solved using a multi-objective algorithm in which the primary objectives were to minimize the drag and the lift (to improve the downforce). To develop the program, the test shape used was an Ahmed Body box that was fixed without any tilt ( 0 degrees relative to the horizontal axis), where the rear surface was defined as five (5) equally spaced points for shape optimization. Alteration of the 5 points were constrained to change within $400 \mathrm{~mm}$ in the positive or negative direction from the reference vertical axis. To achieve optimization, the program was designed to optimize the shape using an Evolutionary Algorithm (EA) - the Elitist Non-Dominated Sorting Genetic Algorithm (NSGA - II). The following parameters were fixed during initialization of the program: number of points for the rear surface, flow velocity, max allowed result. The program began optimization, by generating random candidate surface shapes. Then those candidate solutions were smoothed by the B-Spline function, utilizing applied CFD through the use of OpenFOAM. The program then compared the results and generated new candidate shapes to be tested until 40 generations (for each Reynolds number generated) were produced and the pareto progress was tracked for candidate solution comparison. This resulted in optimized shapes that are similar to experimentally tested and proven shapes that are shaped like a diffuser (as expected) that demonstrated the validity of the program.
\end{abstract}

